\title{
The Effectiveness of Injury-Prevention Programs in Reducing the Incidence of Anterior Cruciate Ligament Sprains in Adolescent Athletes
}

Jeffrey Paszkewicz

Old Dominion University

Tristen Webb

Old Dominion University

Brian Waters

Old Dominion University

Cailee Welch McCarty

Old Dominion University

Bonnie Van Lunen

Old Dominion University, bvanlune@odu.edu

Follow this and additional works at: https://digitalcommons.odu.edu/hms_fac_pubs

Part of the Rehabilitation and Therapy Commons, and the Sports Sciences Commons

\section{Repository Citation}

Paszkewicz, Jeffrey; Webb, Tristen; Waters, Brian; McCarty, Cailee Welch; and Lunen, Bonnie Van, "The Effectiveness of InjuryPrevention Programs in Reducing the Incidence of Anterior Cruciate Ligament Sprains in Adolescent Athletes" (2012). Human Movement Sciences Faculty Publications. 61.

https://digitalcommons.odu.edu/hms_fac_pubs/61

\section{Original Publication Citation}

Paszkewicz, J., Webb, T., Waters, B., McCarty, C. W., \& Van Lunen, B. (2012). The effectiveness of injury-prevention programs in reducing the incidence of anterior cruciate ligament sprains in adolescent athletes. Journal of Sport Rehabilitation, 21(4), 371-377. doi:10.1123/jsr.21.4.371 


\title{
The Effectiveness of Injury-Prevention Programs in Reducing the Incidence of Anterior Cruciate Ligament Sprains in Adolescent Athletes
}

\author{
Jeffrey Paszkewicz, Tristen Webb, Brian Waters, Cailee Welch McCarty, \\ and Bonnie Van Lunen
}

\begin{abstract}
Clinical Scenario: There is a high incidence of anterior cruciate ligament (ACL) injury in adolescents participating in pivoting sports such as soccer, basketball, and handball. Most ACL injuries in athletes are noncontact injuries, with a mechanism of sudden deceleration, change in direction, or landing from a jump. These mechanisms coupled with an increase in contraction of the quadriceps have been shown as risk factors for ACL injuries. Injuries to the ACL may require surgery, a long rehabilitation, and the potential for reinjury. Studies have shown reductions in lower extremity injury rates using training protocols that focus on landing mechanics, balance training, strength training, and/or agility training. There has been some thought that starting preventive training programs with adolescent athletes may be the most effective approach to reducing adolescent ACL injuries. Focused Clinical Question: Can lower extremity injury-prevention programs effectively reduce ACL injury rates in adolescent athletes?
\end{abstract}

Keywords: youth, preventive training program, knee injury

\section{Clinical Scenario}

There is a high incidence of anterior cruciate ligament (ACL) injury in adolescents participating in pivoting sports such as soccer, basketball, and handball. Most ACL injuries in athletes are noncontact injuries, with a mechanism of sudden deceleration, change in direction, or landing from a jump. These mechanisms coupled with an increase in contraction of the quadriceps have been shown as risk factors for ACL injuries. Injuries to the ACL may require surgery, a long rehabilitation, and the potential for reinjury. Studies have shown reductions in lower extremity injury rates using training protocols that focus on landing mechanics, balance training, strength training, and/or agility training. There has been some thought that starting preventive training programs with adolescent athletes may be the most effective approach to reducing adolescent ACL injuries.

\section{Focused Clinical Question}

Can lower extremity injury-prevention programs effectively reduce ACL injury rates in adolescent athletes?

The authors are with the Dept of Human Movement Sciences, Old Dominion University, Norfolk, VA.

\section{Summary of Search, "Best Evidence" Appraised, and Key Findings}

- The literature was searched for studies of level 2 evidence or higher that investigated the effect of injury-prevention training programs on knee-sprain incidence in adolescent athletes.

- The literature search returned 10 possible studies related to the clinical question; 4 studies met the inclusion criteria and were included.

- Two high-quality cluster randomized controlled trials and 2 prospective cohort studies were included.

- Of the included studies, 2 demonstrated reductions of ACL injuries of the intervention group and 2 showed no statistical difference between the intervention group and control group.

\section{Clinical Bottom Line}

There is moderate evidence to support the use of a supervised injury-prevention training program that focuses on developing neuromuscular control of the lower extremity through neuromuscular strengthening exercises, plyometrics, and proprioception exercises. The program should include sessions in the preseason and in-season to reduce the rate of ACL injuries in adolescent athletes. 
Strength of Recommendation: There is grade B evidence that supervised preseason and in-season lower extremity injury-prevention training programs incorporating neuromuscular strengthening exercises, plyometrics, and proprioception exercises are effective in reducing the rate of ACL sprains in adolescent athletes.

\section{Search Strategy}

\section{Terms Used to Guide Search Strategy}

- Patient/Client group: adolescent or youth

- Intervention/Assessment: prevention or prevention program

- Comparison: intervention

- Outcome: ACL injury or injury and incidence or risk

\section{Sources of Evidence Searched}

- The Cochrane Library

- PEDro Database

- Medline

- CINAHL

- SPORTDiscus

- Additional resources obtained via review of reference lists and hand search

\section{Inclusion and Exclusion Criteria}

\section{Inclusion Criteria}

- Level 2 evidence or higher

- Studies investigating the overall rate of ACL injury as the primary objective outcome measure

- Subjects who were actively participating in sport at the time of the study

- Limited to English language

- Limited to humans

- Limited to the past 10 years (2002-2011)

\section{Exclusion Criteria}

- Participants more than 18 years of age

- Studies that did not report specific details of the intervention protocol

- No use of a control group

- Studies using protective equipment as part of the intervention

\section{Results of Search}

Four relevant studies ${ }^{1-4}$ were located and categorized as shown in Table 1 (based on Levels of Evidence, Centre for Evidence-Based Medicine, 2009).
Table 1 Summary of Study Designs of Articles Retrieved

\begin{tabular}{llll}
\hline $\begin{array}{l}\text { Level of } \\
\text { evidence }\end{array}$ & $\begin{array}{l}\text { Study } \\
\text { design }\end{array}$ & $\begin{array}{l}\text { Number } \\
\text { located }\end{array}$ & Reference \\
\hline $1 \mathrm{~b}$ & $\begin{array}{l}\text { Cluster } \\
\text { randomized } \\
\text { controlled trial }\end{array}$ & 2 & $\begin{array}{l}\text { Steffen et al } \\
\text { Olsen et } \mathrm{al}^{2}\end{array}$ \\
\hline $\mathrm{b}$ & $\begin{array}{l}\text { Prospective } \\
\text { cohort }\end{array}$ & 2 & $\begin{array}{l}\text { Pfeiffer et } \mathrm{al}^{3} \\
\text { Mandelbaum }^{3}\end{array}$ \\
& & et al \\
\hline
\end{tabular}

\section{Best Evidence}

The studies in Table 2 were identified as the best evidence and selected for inclusion in this clinically appraised topic (CAT). These studies were selected because they were graded with a level of evidence of 2 or higher, they examined an injury-prevention training intervention in adolescent athletes, and the effect of the intervention on the outcome of interest (rate of ACL injuries) was described.

\section{Implications for Practice, Education, and Future Research}

Based on this appraisal, two ${ }^{2,4}$ of the four ${ }^{1-4}$ studies displayed a significant reduction in ACL injury risk. None of the 4 studies showed an increased risk of ACL injury after the preventive training program. The findings indicate that preventive training programs that focus on developing neuromuscular control of the lower extremity are moderately effective in reducing the risk of ACL sprains in adolescent athletes. The designs of the preventive training programs in each study differed in specific points of emphasis, including implementation of strengthening exercises, plyometrics, balance, and participant compliance in completing the required sessions.

The cluster randomized controlled trial ${ }^{2}$ that demonstrated a significant reduction in ACL injuries used a preventive training program that incorporated a warmup, balance exercises, plyometric exercises, strength and power exercises, and instruction emphasizing technique in planting, cutting, and jumping balance. The cohort study ${ }^{4}$ that demonstrated a significant reduction in ACL injuries used a preventive training program that incorporated a warm-up, stretching, strengthening exercises, plyometric exercises, and sport-specific agility. The cluster randomized controlled trial ${ }^{1}$ that did not report a decrease in ACL injury rate used a preventive training program that included core stability, balance exercises, plyometric exercises, and strengthening exercises. The other cohort study ${ }^{3}$ that did not find a reduction in ACL sprains used a preventive training program that incorporated a progression of plyometric exercises and agility. Even though all 4 of the studies were performed in a 


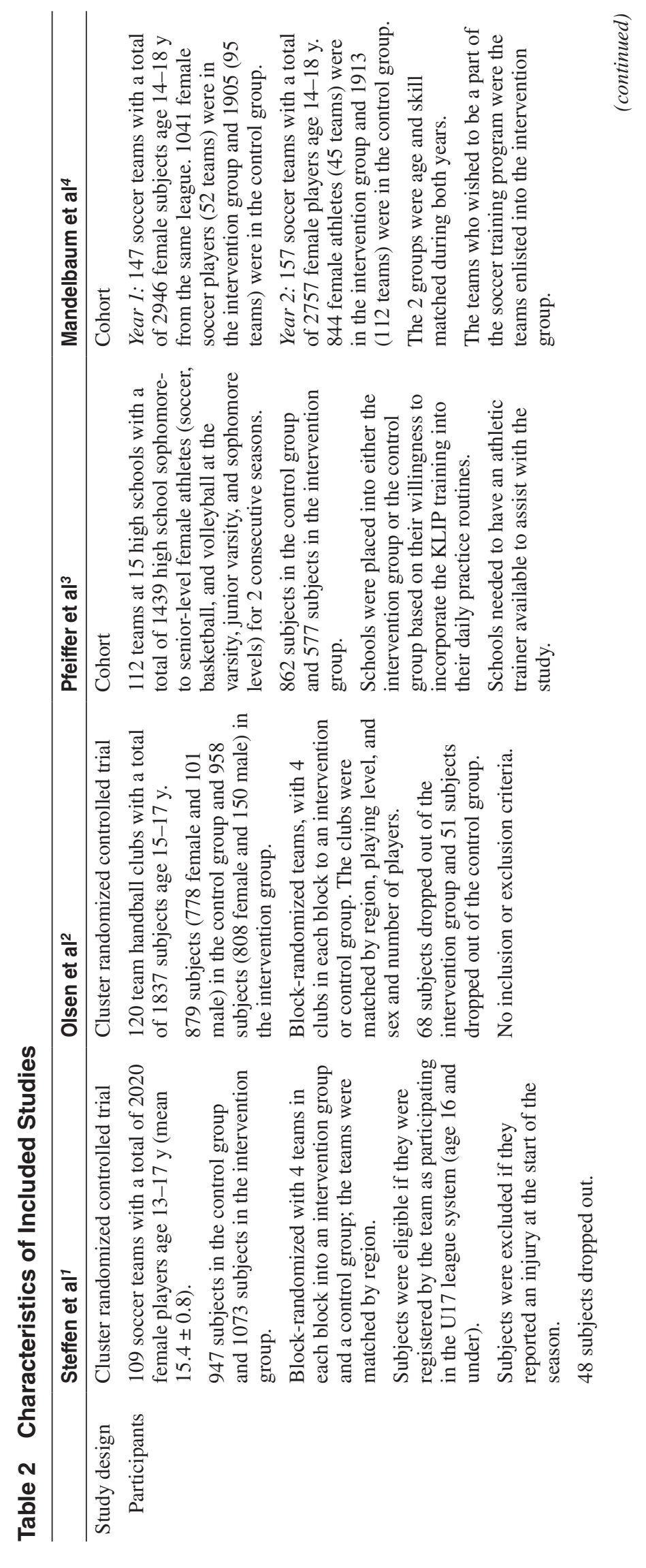




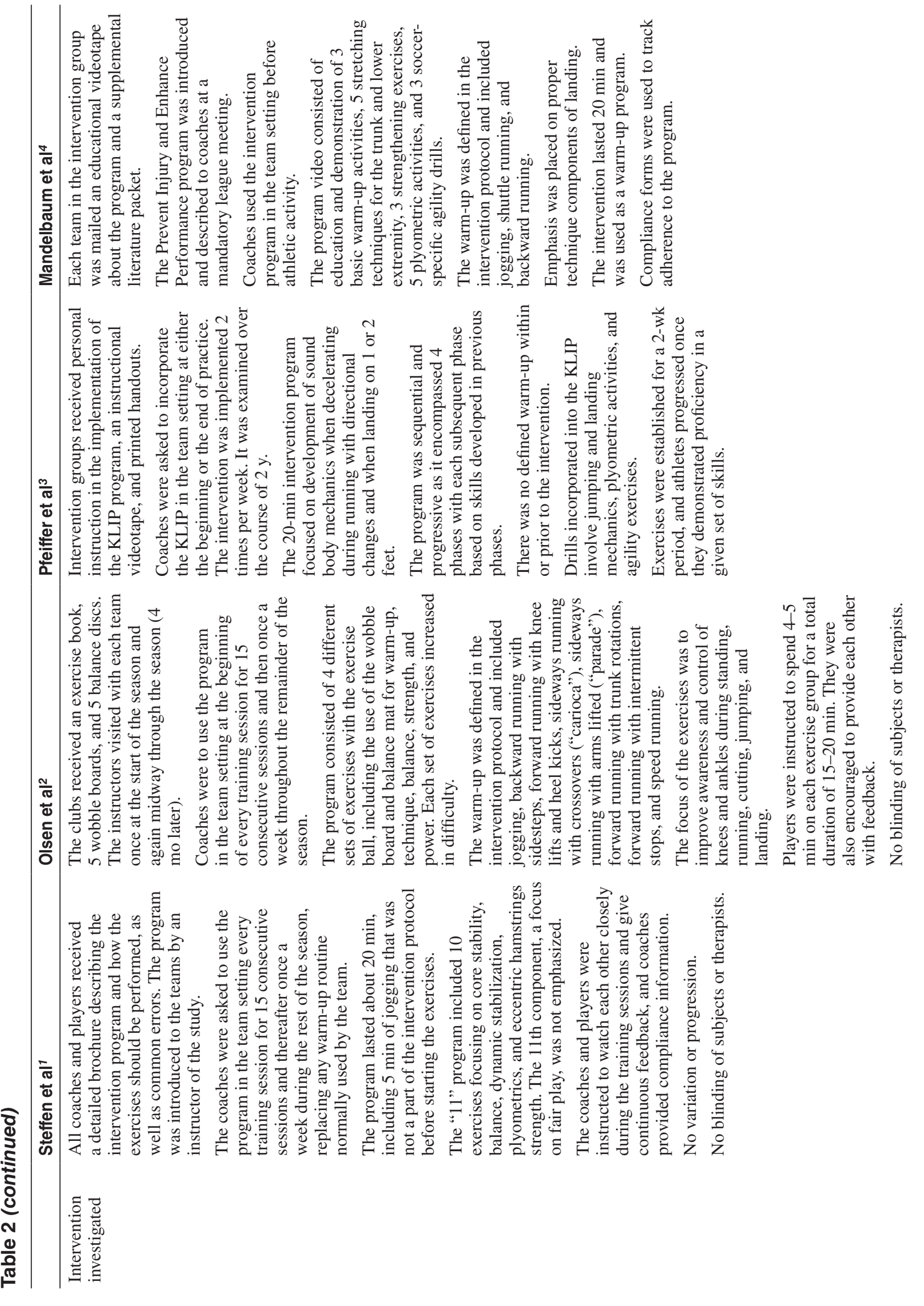




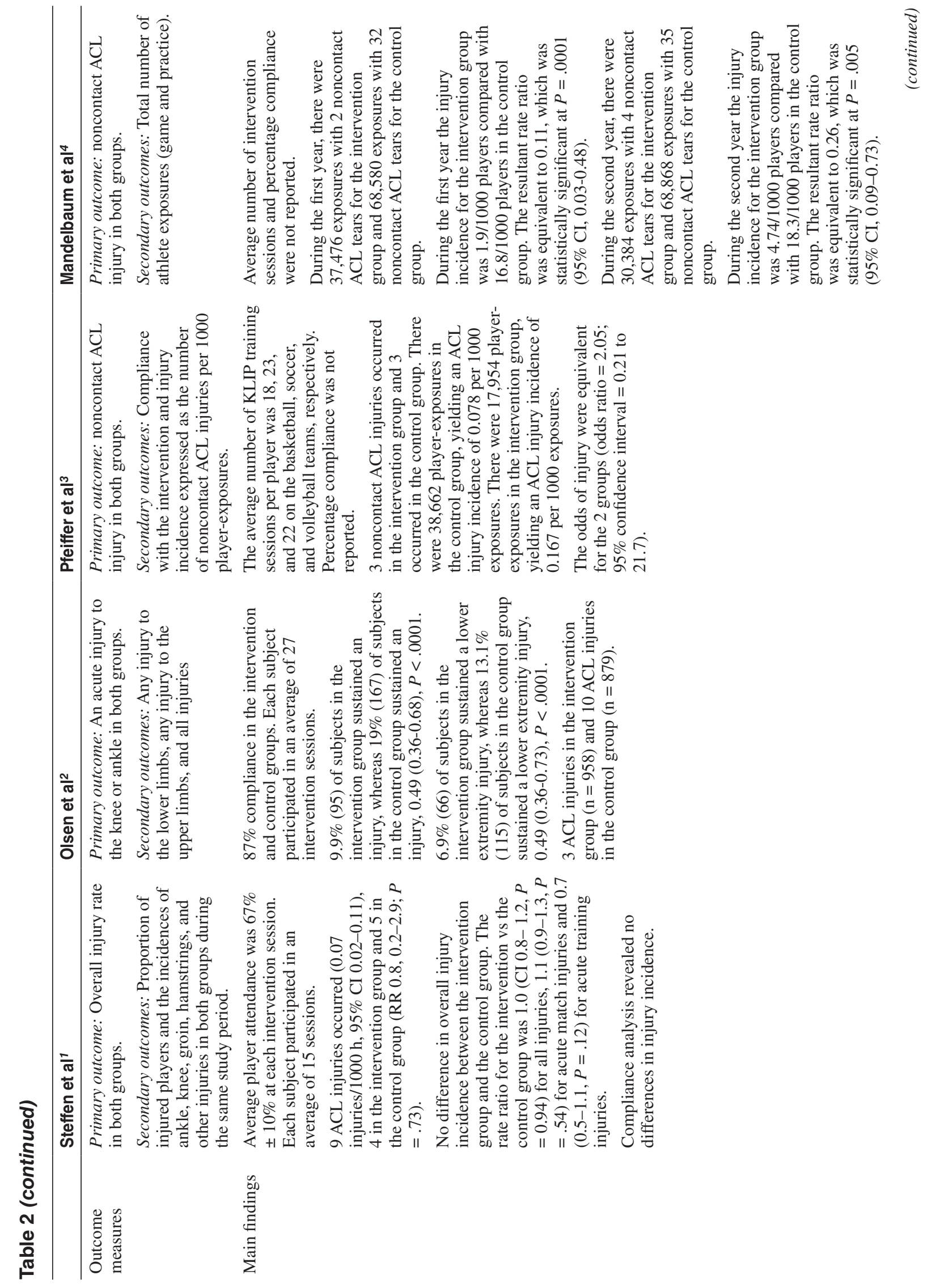




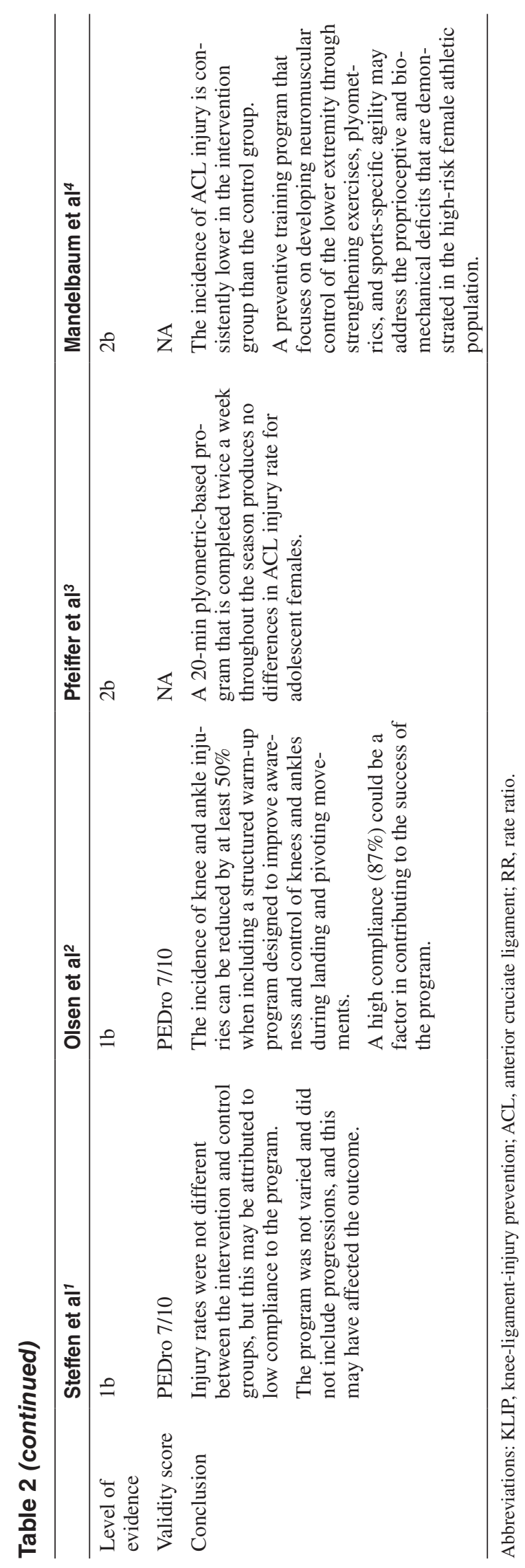


team setting as part of the regular training or practice session, lasted about 20 minutes, and were supervised by a coach, the 2 studies ${ }^{2,4}$ that demonstrated significant reductions in ACL injuries incorporated a standardized warm-up consisting of defined running tasks within the intervention protocol.

Adolescent ACL-injury-prevention programs should consider a supervised preseason phase and in-season phase that incorporates a dynamic warm-up, stretching exercises, lower extremity strengthening exercises, plyometrics, balance, and sport-specific agility with emphasis on proper landing technique stressing a "soft landing" and deep hip and knee flexion ${ }^{2}$. Furthermore, it is important to consider progressions in exercises throughout the duration of the program. Clinicians and coaches should stress to the adolescent athletes that participating in each session is important, and their compliance will be helpful in preventing injury. ${ }^{1,2}$ Supervised injury-prevention programs are easy to incorporate into a warm-up before structured activity and can be incorporated at a low cost to the clinician and team.

Future research regarding adolescent ACL-injuryprevention programs should include well-designed prospective randomized controlled trials that allow for blinding of the subjects and assessors to improve study quality, ${ }^{1-4}$ longer subject follow-up periods, and longer intervention periods that extend over consecutive years. ${ }^{1,3,4}$ Although the incorporation of a standardized warm-up was associated with reductions in ACL injury, ${ }^{2,4}$ other key factors such as participant compliance with the intervention protocol may also be a factor in reduction of ACL injuries. More prospective studies over a longer period of time while tracking participant compliance are needed to determine if the intervention is effective at decreasing ACL injury rate in adolescent athletes. ${ }^{1,2}$ Future research should also investigate the effect of similar aspects of ACL-injury-prevention training programs (ie, strengthening, balance, plyometrics, stretching, and agility) on ACL injury rates between adolescent males and females and compare variations (different sets, repetitions, frequencies, duration, and exercises) to determine the most effective ACL-injuryprevention training program. This CAT should be reviewed in 2 years or when additional best evidence becomes available to determine whether additional information has been published that may change the clinical bottom line for the research question posed in this review.

\section{References}

1. Steffen K, Myklebust G, Olsen OE, Holme I, Bahr R. Preventing injuries in female youth football-a clusterrandomized controlled trial. Scand J Med Sci Sports. 2008;18:605-614.

2. Olsen O, Myklebust G, Engebretsen L, Holme I, Bahr R. Exercises to prevent lower limb injuries in youth sports: cluster randomized controlled trial. BMJ. 2005;330:449452.

3. Pfeiffer RP, Shea KG, Roberts D, Grandstrand S, Bond L. Lack of effect of a knee ligament injury prevention program on the incidence of noncontact anterior cruciate ligament injury. J Bone Joint Surg Am. 2006;88(8):1769-1774.

4. Mandelbaum BR, Silvers HJ, Watanabe DS, et al. Effectiveness of a neuromuscular and proprioceptive training program in preventing anterior cruciate ligament injuries in female athletes: 2-year follow-up. Am J Sports Med. 2005;33:1003-1010. 